\title{
La experiencia docente del uso de objetos de aprendizaje en materias de ingeniería ambiental: el transporte de contaminantes en medios naturales.
}

\section{P. Amparo López Jiménez}

Departamento de Ingeniería Hidráulica y Medio Ambiente. Universitat Politècnica de València. palopez@upv.es

\begin{abstract}
Teaching tools through distance (e-learning) using Internet resources platforms is becoming increasingly important. The reasons for this are several: first, students find that the teaching-learning process can be adapted to your requirements of time and understanding of concepts; and secondly, the possible interactivity. In this regard, the experiences of the author in the development of Learning Objects for interactive teaching in the field of environmental engineering are presented in this contribution. Experience in teaching of subjects in the curriculum field of environmental engineering at second and third cycle over the last two decades by the author has allowed learning objects are prepared with an important follow from that are online. The objects presented in the field of dispersion of pollutants in air and water has allowed students UPV degrees and higher level, find materials to supplement their teaching and value highly the dictates content. This contribution describes these experiences and how students have responded to them.
\end{abstract}

Keywords: Tele-teaching, learning objects, asynchronous teaching, environmental engineering, dispersion of pollutants.

\section{Resumen}

La docencia a través de herramientas a distancia (e-learning) utilizando recursos en plataformas de Internet es cada día más importante. Las razones 
La experiencia docente del uso de objetos de aprendizaje en materias de ingeniería ambiental: el transporte de contaminantes en medios naturales

para ello son varias: de un lado, los alumnos se encuentran con que el proceso enseñanza- aprendizaje puede adaptarse a sus requerimientos de tiempo y comprensión de los conceptos; y de otro lado, la posible interactividad favorecida por este tipo de tecnologías. En este sentido, en esta contribución se presentan las experiencias de la autora en el desarrollo de los Objetos de Aprendizaje para la docencia interactiva en el campo de la ingeniería ambiental. La experiencia en impartición de materias en el campo curricular de la ingeniería ambiental en segundo y tercer ciclo a lo largo de las últimas dos décadas por parte de la autora ha permitido que se preparen objetos de aprendizaje con un seguimiento importante desde que se encuentran on-line. Los objetos descritos en el campo de la dispersión de contaminantes en aire y agua ha permitido que alumnos de titulaciones de la UPV y de un ámbito más amplio, encuentren materiales para complementar su docencia y valoren muy positivamente los contenidos dictados. La presente contribución describe estas experiencias y cómo los alumnos han respondido ante las mismas.

Palabras clave: Teledocencia, objetos de aprendizaje, docencia asíncrona, ingeniería ambiental, dispersión de contaminantes

\section{Introducción}

El uso de herramientas de teleformación en los procesos de aprendizaje es una práctica común y que está dando muy buenos resultados en el émbito de la formación univerisitaria. Así, las experiencias desarrolladas por la autora del presente trabajo se centran en el desarrollo de los llamados Objetos de Aprendizaje para la docencia interactiva en el campo de la fomación ambiental en dispersión de contaminantes en medios fluidos, tanto en agua como en aire.

Los objetos de aprendizaje son videos cortos de entre cinco y diez minutos en los que el profesor desarrolla una idea a través de una explicación y una presentación: En este objeto, a su vez, pueden incluirse modelos numéricos, otros vídeos o enlaces diversos. En particular en el campo de la modelación ambiental de dispersión de contaminantes en medios fluidos esto es particularmente importante, porque interaccionan aspectos de la mecánica de fluidos (con el movimiento del aire); conceptos climáticos con el análisis de las rosas de vientos; o de cosntrucción como la caracterización de chimeneas o de emisarios submarinos, entre otros. Así, pues, el que los alumnos dispongan de estas grabaciones,

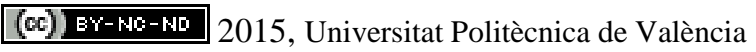

Congreso IN-RED (2015) 
donde, con cierto detalle y pudiendo llamar a otra bibliografía o enlaces, puedan asimilar estos conceptos, se convierte en una herramienta complementaria muy útil para su formación. Esta docencia "on-line" en que el alumno distribuye su propio tiempo y se conecta con los contenidos de los programas de manera atemporal, fuera del instante en que han sido dictados es lo que se reconoce como docencia asíncrona, dentro de la cual se encuentran los objetos de aprendizaje que aquí se describen

El uso de las nuevas tecnologías en la teleformación es una herramienta ya conocida (Garrison, 1998) y con resultados muy prometedores, creciente conforme lo hacen las tecnologías de la información. Los objetos de aprendizaje son herramientas docentes con muchas posibilidades, susceptibles de ser integrados en programas multidisciplinares con docencia síncrona (de forma que los alumnos pueden interactuar con el profesor a través de foros en ciertas horas abiertas de tutorías); en ocasiones, como docencia inversa (cuando los alumnos se preparan el temario a través de tele formación como la que se presenta y acuden a clase solamente para realizar aplicaciones de los conceptos que se han podido trabajar a distancia); o finalmente como docencia asíncrona: cuando los materiales se dejan en internet y a través del enlace correspondiente, los alumnos reciben los conceptos fundamentales. Las dudas y talleres finalmente se acaban debatiendo en un foro, desde el momento en que todos los alumnos han visualizado los videos y presentaciones..

La apertura de estos materiales a foros internacionales siempre le da un cariz muy positivo a la retroalimentación que se conoce de la docencia impartida, como se ha comprobado por la autora, con la gran cantidad (y calidad) de consultas desde otros países. Las experiencias en este campo se han documentado en el marco de estudios de últimos ciclos en la Universidad española ya en la última década (Onrubia et al, 2006).

La puesta en valor de la preparación de elementos que favorezcan el autoaprendizaje basado en tecnologías de la información no es nueva, ya en 2000, en el Consejo Europeo de Lisboa, la Comisión Europea presentó un plan de acción con nombre e-Learning, para la educación a distancia. Encuadrado dentro del plan e-Europe, e-Learning, entre otras presenta las siguientes directrices para su seguimiento:

- Garantizar que todos los alumnos tengan derecho a una formación digital.

- Integrar en una formación adecuada al aprendizaje a distancia para los docentes

- Favorecer que los egresados tengan la posibilidad de tener una cultura digital a través del aprendizaje permanente.

Desde entonces, estas directrices no han dejado de tener vigencia (COM/2013/0654 final).

Es importante desarrollar estrategias atractivas y motivantes que cubran esta necesidad que la sociedad presenta y la Comunidad Europea enuncia, para conseguir en todos los ámbitos universitarios que los alumnos encuentren herramientas de complemento para su formación

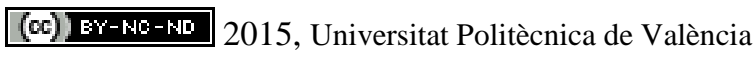

Congreso In-Red (2015) 
La experiencia docente del uso de objetos de aprendizaje en materias de ingeniería ambiental: el transporte de contaminantes en medios naturales

a través de plataformas de Internet. Esto es especialmente interesante en el último tramo de la educación superior, cuando los alumnos se supone que son más autónomos. Es asimismo, fundamental que el profesor o tutor que desarrolla los objetos, sea un participante activo en este proceso, preparando documentos y estando atento a los foros, de manera que el proceso de aprendizaje se mantenga vivo. El profesor que prepara y presenta los objetos juega a continuación un papel de "tutor a distancia" de alumnos a los que no conoce, que consultan los materiales de forma concreta, jugando un rol de apoyo temporal que motive y facilite a los alumnos, en este espacio virtual, que éstos mejoren su nivel de aprendizaje, más allá de las competencias normales de habilidades que poseen y que reciben en su docencia presencial (Pagano, 2007).

En este sentido, desde la Universitat Politècnica de València, se ha potenciado la creación de los llamados "Objetos de aprendizaje" ya descritos. Un objeto de aprendizaje se define como "la unidad mínima de aprendizaje, en formato digital, que puede ser reutilizada". Para que la reutilización sea posible es imprescindible que el objeto no esté contextualizado: No debe hacerse referencia a un momento concreto del curriculum del alumno, de forma que se amplía mucho la aplicabilidad de este objeto. Estas herramienas son de gran ayuda como herramientas de motivación al dotar al alumno de libertad de horario y no tener que desplazarse, siendo en si mismo una alternativa motivadora para el alumno, al que dota de una mayor libertad (Alomonte, Ed. 2012). Así, los objetos de aprendizaje cumplen con sobrada solvencia sus intenciones.

\section{Objetivos}

Lograr que los alumnos de ingeniería conozcan los conceptos de dispersion de contaminantes, involucra principalmente análisis numéricos y basados en ecuaciones, difíciles de transmitir a los alumnos. Para hacer llegar estos conocimientos técnicos a los estudiantes, se hacen muy interesante el uso de tecnologías amenas de comunicación y simulaciones gráficas o presentaciones que permitan a los alumnos asimilar los conocimientos en repetidas ocasiones y a un ritmo mucho más pausado de lo que la clase magistral en ocasiones permite (Edith y Alvarado, 2014).

En este sentido, el desarrollo de los objetos de aprendizaje en el caso particular de la dispersión de contaminantes que nos ocupa en la presente comunicación, se ha centrado en la preparación de unos objetos clave para que los alumnos adquieran los conocimientos y motivación en este campo. Se entiende que el enfoque, de un lado amplio al ser temas de índole multidisciplinar; y de otro lado concreto, al detallar aspectos particulares como el diseño de chimeneas o emisarios submarinos, puede suponer un elemento diferencial que 
haga a los alumnos consultarlos como complemento a sus diferentes formaciones de origen. Así pues, se ha considerado que los objetos a desarrollar son los siguientes:

- La modelación de la calidad del agua. El objetivo es que los alumnos conozcan las diferentes estrategias de las que se dispone en relación con la modelación computacional para determinar la calidad del agua en fuentes de suministro, a lo largo del sistema de abastecimiento; o bien en aguas superficiales. Se introducen las ecuaciones que representan los procesos que alteran la calidad de las aguas y las posibles soluciones que, en forma de programas computacionales, se encuentran a nuestra disposición.

- Los emisarios submarinos: El objetivo es que los alumnos conozcan el emisario submarino como final del sistema de saneamiento y los objetivos de dispersión que tiene que cumplir. Se introducen las partes del mismo y las metodologías de cálculo para realizar un diseño prelimina

- Calidad de aguas, las fuentes de suministro. El objetivo es que los alumnos adquieran un conocimiento de las fuentes de suministro del agua, crucial para un correcto análisis de la red de abastecimiento y en aras de la sostenibilidad, para conocer todas las posibilidades de disponer de agua potable.

- Modelo Gaussiano de dispersión de contaminantes. La dispersión de contaminantes atmosféricos es una materia que responde a complicadas ecuaciones para ser analizada. El presente introduce a los procesos por los cuales se dispersan los contaminantes en la atmósfera y la manera en que un modelo analítico sencillo puede suponer (en ocasiones en que sus simplificaciones son aplicables); una solución adecuada para conocer las concentraciones de diversos contaminantes tanto en la atmósfera como a nivel de suelo.

- El proceso de dispersión de contaminantes en medios receptores. El objetivo del objeto de aprendizaje es que los alumnos conozcan de forma cualitativa los fenómenos que determinan este transporte y dispersión de contaminantes en medios fluidos naturales, cuáles son los principios físicos que los definen y la forma de prever las concentraciones finales a través de la modelación.

- La contaminación atmosférica de origen antropogénico. En el presente objeto el lumno aquiere el conocimiento de los procesos que determinan esta dispersión de contaminantes y que hacen disminuir la concentración desde la emisión (salida a la atmósfera) hasta la inmisión (llegada al suelo) cuando las contaminaciones se deben a la acción del hombre. 
La experiencia docente del uso de objetos de aprendizaje en materias de ingeniería ambiental: el transporte de contaminantes en medios naturales

- La importancia de las chimeneas en el proceso de dispersión de contaminantes atmosféricos. El presente objeto de aprendizaje pone de manifiesto la importancia que juega el diseño de la propia chimenea en este proceso de dispersión, no realizada a ras de suelo sino a una cierta altura para que los contaminantes lleguen al suelo con una concentración aceptable.

- Determinación del tiro de una chimenea industrial. Al consultar el presente objeto de aprendizaje los alumnos conocerán los fenómenos que intervienen en la determinación de esta capacidad de tiro de las chimeneas para que el flujo de salida de los gases sea suficiente.

\section{Desarrollo de la innovación}

El desarrollo de objetos de aprendizaje como complemento a la formación curricular del alumno es conocido desde hace tiempo (Chan, 2001). La innovación viene de construir objetos de aprendizaje en torno a problemáticas significativas según las orientaciones de las diferentes titulaciones, de forma que pueda adaptarse a muchas de ellas, en el marco del nuevo Espacio Europeo de Educación Superior. Ello supone una vinculación importante entre las funciones de investigación y docencia.

La producción de objetos así, podrán ser orientados al desarrollo de competencias que tengan un planteamiento lo suficientemente abierto como para ser contextualizados por alumnos procedentes de diversidad de ámbitos y se favorezca la motivación del alumno y el conocimiento más profundo y de forma autónoma de las materias descritas.

En este sentido, se considera que se han conseguido los siguientes hitos:

- $\quad$ Se ha potenciado la utilización de medios técnicos, como recurso facilitador del aprendizaje en cuanto a que reduce los obstáculos de carácter geográfico, económico y laboral para que el alumno pueda acceder a la formación específica. Ello hace que las nuevas tecnologías se conviertan en una herramienta para la "democratización del conocimiento", particularmente importante en aspectos ambientales que tienen muchas connotaciones sociales y un muy amplio espectro de aplicación.

- $\quad$ Se ha motivado al alumno a organizar su tiempo y tareas como apoyo a sus clases teóricas. Los alumnos no tienen obligación de trabajar con estos conocimientos. El uso de los objetos debe complementar su formación. Ello hace que el aprendizaje se deba convertir en una actividad flexible, en la que el estudiante marca su propio tiempo y puede profundizar en aquellos temas que hayan despertado más su atención. 
- Se ha potenciado la comunicación bidireccional entre el alumno y el profesor, a través del correo electrónico, de manera que ha servido para poner en contacto a alumnos de muy diversas procedencias con un profesorado local, que presenta problemas globales, como lo es la dispersión de contaminantes en los medios receptores fluidos.

Asimismo, no todo son ventajas. El seguimiento de estos objetos también ha tenido dificultades: los alumnos se encuentran en ocasiones muy solos para organizar su tiempo, con lo que el grado de aprovechamiento depende de la voluntad del estudiante; en ocasiones no se dispone de la capacidad tecnológica adecuada, por ejemplo, para implementar modelos de cálculo que se describen someramente en los contenidos, o la complejidad de los contenidos hacen que el alumno no se motive sobre los contenidos de los mismos, y no considere interesante la implementación de los resultados de este aprendizaje. Sin embargo, estas dificultades deben vencerse poniendo en contacto al alumno con el profesor a través del correo electrónico, de forma que el envío de materiales suplementarios, la respuesta a preguntas concretas o la propuesta de ejercicios de aplicación de los conocimientos adquiridos, haga que el alumno encuentre un sentido a la formación que se propone y redirija su aprendizaje en estos casos concretos.

\section{Resultados}

La autora tiene una gran experiencia preparando objetos de aprendizaje en el marco de los programas de Docencia en Red en la actualidad y de Universitat Politècnica Abierta en el pasado.

En el marco de la ingeniería ambiental y el programa Docencia en Red de la UPV, la autora ha desarrollado ocho objetos de aprendizaje en el ámbito de la dispersión de contaminantes en agua y aire.

Como muestra de entre ellos, uno de los más activos y visitados es el denominado "Modelo Gaussiano de dispersión de contaminantes". Este objeto ha recibido 545 visitas continuadamente desde su creación, de entre ellas solamente 173 son de España. Los resultados concretos en este caso, al no tener una evaluación particular del objeto, deben medirse en forma de variadas consultas que denotan el interés suscitado. La gran disponibilidad de los objetos de aprendizaje en un entorno global y la importancia de los contenidos descritos en este objeto hacen que haya sido causa de numerosas peticiones a la autora por parte de los alumnos de información complementaria y bibliografía que permita profundizar en los conceptos descritos. Ello informa sobre los buenos resultados que ha dado este tipo de objetos de aprendizaje y los que se espera que den en el futuro.

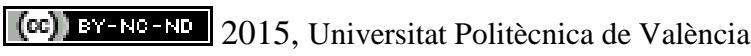

Congreso In-Red (2015) 
La experiencia docente del uso de objetos de aprendizaje en materias de ingeniería ambiental: el transporte de contaminantes en medios naturales

\section{Conclusiones}

La experiencia de la proposición de objetos de aprendizaje en el marco de la ingeniería ambiental y el seguimiento de los mismos desde su creación demuestra la potencialidad eficaz de integración de modelos de docencia a través de plataformas de Internet. Esta integración, relacionando progresivamente los medios tecnológicos y el aprendizaje significativo, revela en cada etapa la posibilidad de representar más sintéticamente los conceptos, y favorece una mayor reflexión por parte del alumno de los conocimientos presentados, fundamental para que los conocimientos se conviertan en perdurables.

El seguimiento de los objetos descritos por parte de alumnos de todo el mundo, favorecido por la transcripción y traducción escrita de los textos dictados, ha favorecido una mayor comprensión de la situación y motivación, según indican alumnos que han seguido los objetos. Se advierte, de esta manera, un recurso expresivo eficaz para una integración pedagógica amplia de los contenidos presentados relativos a la dispersión de contaminantes en el marco de la ingeniería ambiental.

Sin embargo, la propuesta de aprendizaje a través de una presentación online como la que se describe, cuenta con una limitación en el manejo de procedimientos técnicos que requieren ser preparados para la experiencia. Esto es, la elaboración de los contenidos y modelos que puedan describir los procesos de dispersión de contaminantes y su ubicación en los estudios de alumnos de últimos cursos, traslada a los docentes una planificación muy pensada sobre el contenido de las presentaciones. Debe generarse una secuencia narrativa breve y visual ordenada e interesante, antes de proponerla al alumno para su visualización. Por ello, es definitivo para la efectividad de este recurso que las actividades pedagógicas ayuden a los docentes a preparar buenos materiales, apoyados de forma institucional por la entidad que propone los conocimientos, como ocurre en este caso concreto. Los buenos resultados basados en las estadísticas de alumnos que se descargan los objetos continuadamente desde su creación, avalan el interés de los contenidos aquí descritos.

\section{Referencias}

ALOMONTE, S. (Ed.) ( 2012). Educación en Arquitectura Sostenible. Libro blancoResumen ejecutivo. EDUCATE. Environmental Design in University Curricula and Architectural Training in Europe. 
CHAN, M. E. (2001). OBJETOS DE APRENDIZAJE: una herramienta para la innovación educativa. INNOVA. Universidad de Guadalajara, Mexico. 2001

COM/2013/0654 final. 2013. COMMUNICATION FROM THE COMMISSION TO THE EUROPEAN PARLIAMENT, THE COUNCIL, THE EUROPEAN ECONOMIC AND SOCIAL COMMITTEE AND THE COMMITTEE OF THE REGIONS Opening up Education: Innovative teaching and learning for all through new Technologies and Open Educational Resources. Consultado en abril de 2015 en: http://eur-lex.europa.eu/legalcontent/EN/TXT/?qid=1389115469384\&uri=CELEX:52013DC0654

EDITH GRANERO, A.; GARCIA ALVARADO, R. (2014). Aprendizaje temprano de arquitectura sustentable mediante vistas interiores graduadas. Revista Hábitat Sustentable. V4. N1. Junio de 2014. pp 14-24.

GARRISON, D. R. (1998). Andragogy, learner-centeredness, and the educational transaction at a distance. Journal of Distance Education, 3, (2), 123-127.

ONRUBiA, J., BUSTOS, A., ENGEL, A. Y SEGUÉS, T. (2006). Usos de una herramienta de comunicación asíncrona para la innovación docente en contextos universitarios. IV Congreso Internacional de Docencia Universitaria i Innovació. Barcelona . (España).

PAGANO, C.M. (2007). Los tutores en la educación a distancia. Un aporte teórico.» [artículo en línea]. Revista de Universidad y Sociedad del Conocimiento (RUSC). Vol. 4, n. ${ }^{\circ}$ 2. UOC. [Consultada en Abril de 2015 ].

$<$ http://www.uoc.edu/rusc/4/2/dt/esp/pagano.pdf $>$. ISSN 1698-580X 